\title{
Osteogenic potential of cells derived from nasal septum*
}

\author{
Elena Torreggiani ${ }^{1}$, Chiara Bianchini ${ }^{2}$, Letizia Penolazzi ${ }^{1}$, Elisabetta Lambertini ${ }^{1}$, \\ Renata Vecchiatini ${ }^{1}$, Alessandro Canella ${ }^{1}$, Roberto Gambari ${ }^{1}$, Eros Magri ${ }^{3}$, \\ Stefano Pelucchi², Antonio Pastore ${ }^{2}$, Roberta Piva ${ }^{1}$ \\ 1 Department of Biochemistry and Molecular Biology, Section of Molecular Biology, University of Ferrara, \\ Ferrara, Italy \\ 2 Otorhinolaryngology Unit, Department of Medical and Surgical Specialities, University Hospital of Ferrara, \\ Ferrara, Italy \\ 3 Department of Experimental and Diagnostic Medicine, Section of Anatomic Pathology, University of Fer- \\ rara, Ferrara, Italy
}

\begin{abstract}
SUMMARY
Background: The research addressed to detect new molecular targets in the development of therapeutic strategies aimed to repair bone tissues.

The aim of this study was to determine the potential osteogenic activity of bone cells from the nasal septum and their use to perform accurate molecular analysis from a single sample.

Methodology: The cells, after nasal septum surgery, were subjected to gene silencing, Reverse Transcriptase - Polymerase Chain reactions, immunocytochemistry and chromatin immunoprecipitation.

Results: Cells from the nasal septum can give rise to mature osteoblasts that express osteogenic markers (ALP, Runx2, Slug) and are able to mineralize. We demonstrated that Runx2, a transcription factor critical in early osteospecific differentiation, interacts in vivo with the promoter of the SLUG gene, a marker of osteoblast maturation.

Conclusions: We demonstrated that nasal septum-derived osteoblasts represent an interesting alternative source for bone forming cells, and a promising material to be utilized in bone cellular therapy.
\end{abstract}

Key words: human primary osteoblasts, nasal septum, chromatin immunoprecipitation, bone repair

\section{INTRODUCTION}

There is currently no ideal approach for the treatment of craniofacial defects. Such defects arise from trauma, injuries, removal of bone tissue as a result of cancer, and developmental abnormalities. They often lead to massive destruction of the facial skeleton ${ }^{(1-4)}$. Tissue engineering, which is based on the use of a combination of appropriate cells, growth factors, engineering methods, and suitable carrier scaffolds to improve or replace biological functions, promises to offer new therapeutic chances for the repair of damaged bone ${ }^{(4-6)}$. Bone tissue engineering currently uses either growth factors on a variety of carriers or osteogenic cells seeded onto an even larger number of different materials ${ }^{(4-7)}$. In the craniofacial area, despite a decade of experimental evaluation, ambiguous results have been obtained in the reconstruction of bony defects, and a large number of unresolved questions remain in the head and neck area ${ }^{(7-17)}$. This is correlated with evidence that surgical repair of such defects, as well as three-dimensional enlargement of structures in the facial skeleton are remarkable difficult and unpredictable. Particularly, complex bony defects are difficult to reconstruct accurately, and complications in the area of reconstruction are common. It has been demonstrated that many unsatisfactory results are correlated with poor cell survival rate, and inferior bone specific cellular activity after transplantation has to be considered as reason of occurance due to premature differentiation and subsequent growth inhibition ${ }^{(18)}$. In addition, the extremely high dosages of growth factors that are required to successfully regenerate bone in a clinically relevant dimension is to some extent controversial. Therefore, because biomolecules, as well as cells, both embryonic and adult tissue derived, play a critical role in this field of research, a multidisciplinary approach is absolutely required for a successful outcome.

Based on of these considerations, we investigated the characteristics of bone cells from the nasal septum in terms of potential osteogenic activity and experimental models to perform an accurate molecular analysis for detection of new therapeutic targets. We demonstrate here that these cells represent an interesting alternative source for bone forming cells. They can be an ideal, 
unique, easily accessible and no controversial source for osteoblasts, due to the simple collection procedure during nasal septum surgery once bone tissue is routinely discharged. The ability to proliferate and to differentiate into mature osteoblasts at the same time makes the use of these cells particularly attractive both for the research addressed to new molecular targets detection, and the development of therapeutic strategies aimed to repair and replace damaged tissues. In addition, we demonstrated that, from a single sample, it is possible to obtain sufficient material to perform several molecular analyses including gene expression studies by Reverse Transcriptase - Polymerase Chain Reaction (RT-PCR), DNA-protein interactions assays by chromatin immunoprecipitation (ChIP), and immunocytochemical experiments.

\section{MATERIALS AND METHODS}

Isolation and culture of osteoblasts

Human craniofacial osteoblast cells were obtained from bone samples collected during nasal septum surgery. Recruitment of subjects donating osteoblasts was in accordance with approved procedures, and informed consent was obtained from each patient. Bone samples were collected from 81 patients $(30 \mathrm{~F} / 51 \mathrm{M})$ that underwent septoplasty surgery, FESS or both procedures (for 8 cases it was possible to collect osteoblasts both from septum and ethmoid bone) from June 2006 to February 2010 at the ENT Department of the University Hospital of Ferrara. This type of surgery is routinely and worldwide performed in an ENT Surgery Unit. It usually consists in the exeresis of the lower part of the osseous nasal septum (this portion has been used in this study). This sampling is very handily and costless. The average age of patients was 43 years (range: $22-75$ ). Ten out 81 samples were selected and dedicated to the studies here presented. Briefly, the bone samples, about $1 \mathrm{~cm}^{2}$ in size, were cut into small fragments that were washed several times in phosphate-buffered saline (PBS) to remove blood cells and debris with a final wash in culture medium. Fragments were then collected in culture flasks containing $6 \mathrm{ml}$ of 1:1 mixture of DMEM/Ham's F12 (Sigma Aldrich, St. Louis, MO, USA) supplemented with $20 \%$ fetal bovine serum (FBS) (CELBIO EuroClone, Milan, Italy), $2 \mathrm{mM}$ glutamine, 100 units $/ \mathrm{ml}$ penicillin, $100 \mathrm{mg} / \mathrm{ml}$ streptomycin, and $50 \mathrm{mg} / \mathrm{ml}$ ascorbate at $37^{\circ} \mathrm{C}$ in a humidified atmosphere of $5 \% \mathrm{CO}_{2}$. After about 5-7 days, outgrowth of bone cells from the bone chips commenced, and confluency in $25 \mathrm{~cm}^{2}$ dishes was usually reached after 3-4 weeks ${ }^{(19)}$. For the studies here presented, only first and second passage cells were used.

\section{Flow cytometric analysis}

The cells from all 10 samples were analysed for expression of surface marker molecules, by direct immunofluorescent staining. Briefly, cell pellets were resuspended in PBS and incubated with fluorescein isothiocyanate (FITC)- or phycoerythrin (PE)- conjugated mouse anti-human antibodies Stro1, CD45PE, and CD90-FITC (DakoCytomation, Dako, Denmark), for $15 \mathrm{~min}$ at $4^{\circ} \mathrm{C}$. Monoclonal antibodies with no specificity were used as negative control. Antibody treated cells were then washed with PBS and spinned down. For each sample, the cell pellet was resuspended in $400 \mu \mathrm{l}$ of PBS and the fluorescence of 20,000 cells was analysed using FACS Scan flow cytometer (Becton Dickinson, New Jersey, USA) and CellQuest software (Becton Dickinson European HQ, Erembodegem Aalst, Belgium). Flow cytometric analysis was repeated three times for each sample to allow SEM calculation.

\section{Analysis of the osteoblast phenotype}

For alkaline phophatase staining, prefixed mono-layered cells from all 10 samples were incubated at room temperature in a solution containing naphthol AS-BI phosphate and freshly prepared fast blue BB salt buffered at $\mathrm{pH} 9.5$ with 2-amino-2methyl-1.3-propanediol (Alkaline Phosphatase Leukocyte kit, Sigma). The presence of sites of ALP activity appeared as blue cytoplasmatic staining.

The extent of mineralized matrix in the plates was determined by Alizarin Red S staining (Sigma) in the cells cultured for up to 35 days in osteogenic medium consisting in DMEM, highglucose, supplemented with $10 \% \mathrm{FBS}, 10 \mathrm{mM}$ b-glycerophosphate, $0.1 \mathrm{mM}$ dexamethasone and $50 \mathrm{mM}$ ascorbate. In the committed cells, the osteogenic medium was changed every 3 days. The cells were then fixed in $10 \%$ formaldehyde for $15 \mathrm{~min}$ at room temperature, washed with deionized water, stained with $40 \mathrm{mM}$ AR-S (pH 4.2) for $20 \mathrm{~min}$ at room temperature, and washed 5 times with deionized water to eliminate non-specific staining. The stained matrix was observed at different magnifications using a Leitz microscope.

\section{Immunocytochemistry}

Immunocytochemistry analysis was performed on all 10 samples using an ImPRESS Universal Reagent Kit (Vector Laboratories, Inc. Burlingame, CA, USA). About $10^{4}$ cells were seeded in 4-well chamber slides, led to adhere for $48 \mathrm{hrs}$, fixed in cold $100 \%$ methanol and permeabilised with $0.2 \%$ (vol/vol) Triton X-100 (Sigma Aldrich) in TBS (Tris-buffered saline). Cells were incubated in $0.3 \% \mathrm{H}_{2} \mathrm{O}_{2}$ and the endogenous peroxidase was blocked with ready-to-use $(2.5 \%)$ normal horse blocking serum (ImPRESS Reagent Kit, Vector Laboratories). Afterwards, the primary antibodies, two polyclonal antibodies against human Runx2 (M-70; 1:100 dilution) and human Slug (H-140; 1:100 dilution) (Santa Cruz, Biotechnology, CA, USA), respectively, were applied and incubated at $4^{\circ} \mathrm{C}$ overnight. Cells were then incubated at room temperature with ImmPRESS reagent (ImPRESS Reagent Kit) for $30 \mathrm{~min}$. After rinsing in TBS, substrate-chromogen mix (ImmPACT DAB, Vector Laboratories). After washing, cells were mounted in glycerol/PBS 9:1 and observed using a Leitz microscope.

\section{Small interfering RNA (siRNA) transfection}

BLOCK-iT Fluorescent Oligo (Invitrogen, Carlsbad, CA, USA) was used to assess the transfection efficiency of siRNA. It is a fluorescein-labelled, double-stranded RNA duplex with the same length, charge and configuration as standard siRNA. The sequence of the BLOCK-iT Fluorescent Oligo is not 
homologous to any known gene, ensuring against nonspecific cellular events caused by the introduction of the oligonucleotide into the cells. Human osteoblasts were seeded under the same experimental conditions and transfected with $30 \mathrm{nM}$ of BLOCK-iT Oligo. After 24 hours, images of transfected cells were recorded using a digital imaging system based on a fluorescence microscope (model Axiovert 200L; Carl Zeiss MicroImaging, Inc.) equipped with a back-illuminated CCD camera (Roper Scientific), excitation and emission filterwheels (Sutter Instrument Company), and piezoelectric motoring of the $\mathrm{z}$ stage (Physik Instrumente, $\mathrm{GmbH} \& \mathrm{Co}$ ). The data were acquired and processed using the MetaFluor analyzing program (Universal Imaging Corp).

Stealth RNAi duplexes and corresponding Stealth control were synthesized by Invitrogen Life Technologies. Stealth RNAi compounds are 25 mer dsRNA containing proprietary chemical modifications that enhance nuclease stability and reduce off-target effects. The previously described siRNA/ Slug2 targeting human Slug was used ${ }^{(20)}$. Twenty four hrs before siRNA transfection, hOBs were seeded in triplicate at a density of $16 \times 103 / \mathrm{cm}^{2}$ in DMEM with $10 \%$ FBS. Cells were transfected with $30 \mathrm{nM}$ siRNA using Lipofectamine RNAiMAX (Invitrogen Life Technologies) according to the manufacturers' instructions. Transfected cells were incubated for 6 days at $37^{\circ} \mathrm{C}$ before gene silencing analysis. As a negative control for the siRNA treatment, Medium GC Stealth RNAi Negative Control Duplex (Invitrogen) was used. Knockdown of Slug expression was verified by Real-Time RT-PCR.

\section{Real-time quantitative RT-PCR}

Cells from three wells were harvested and total RNA was extracted using an RNeasy Mini Kit (Qiagen GMBH, Hilden, Germany) according to the manufacturer's instruction and as previously described ${ }^{(20)}$. Real-time PCR was carried out using the ABI PRISM 7700 Sequence Detection System (Applied Biosystems Inc, Foster City, CA, USA), TaqMan technology, and the Assays-On-Demand kit for human Slug, Runx2, and BSP. The mRNA levels of target genes were corrected for GAPDH mRNA levels (endogenous control). All PCR reactions were performed in triplicate for each sample and were repeated three times. All experimental data were expressed as the mean \pm S.E.M.

\section{Western blotting}

For western blot analysis, the cells were washed twice with icecold PBS and cell lysates were prepared as previously reported (19). Then, $10 \mu \mathrm{g}$ of each sample was electrophoresed through a $12 \%$ SDS-polyacrylamide gel. The proteins were then transferred onto an Immobilon-P PVDF membrane (Millipore, Billerica, MA, USA). After blocking with PBS - 0.05\% Tween 20 and $5 \%$ dried milk, the membrane was probed with the following antibodies: Slug (L40C6) from Cells Signaling Technology (Danvers, CA, USA), and IP3 K (06-195) from Upstate Biotechnology (Lake Placid, NY, USA). After washing with PBS-Tween, the membranes were incubated with peroxidase-conjugated anti-rabbit antibody (1:50000) or anti- mouse (1:2000) (Dako) in 5\% non-fat milk. Immunocomplexes were detected using Supersignal West Femto Substrate (Pierce, Rockford, IL, USA). Anti-IP(3)K was used to confirm equal protein loading.

\section{Chromatin immunoprecipitation (ChIP) assay}

The ChIP assay was carried out as previously described using the standard protocol supplied by Upstate Biotechnology, Inc. with their ChIP assay reagents ${ }^{(21)}$. The cells were cross-linked with $1 \%$ formaldehyde for $10 \mathrm{~min}$ at $37^{\circ} \mathrm{C}$, washed in ice-cold PBS and suspended in SDS lysis buffer for $10 \mathrm{~min}$ on ice. Samples were sonicated, diluted 10 -fold in dilution buffer supplemented with protease inhibitors and precleared with $80 \mathrm{ml}$ of DNA-coated protein A-agarose; the supernatant was used directly for immunoprecipitation with $5 \mu \mathrm{g}$ of anti-Runx2, (sc-10758) (Santa Cruz Biotec), overnight at $4{ }^{\circ} \mathrm{C}$. Immunocomplexes were mixed with $80 \mathrm{ml}$ of DNA-coated protein A-agarose followed by incubation for $1 \mathrm{hr}$ at $4^{\circ} \mathrm{C}$. Beads were collected and sequentially washed 5 times with 1 $\mathrm{ml}$ each of the following buffers: low salt wash buffer $(0.1 \%$ SDS, $1 \%$ Triton X-100, 2 mM EDTA, $20 \mathrm{mM}$ Tris- $\mathrm{HCl} \mathrm{pH}$ $8.1,150 \mathrm{mM} \mathrm{NaCl})$, high salt wash buffer $(0.1 \% \mathrm{SDS}, 1 \%$ Triton X-100, 2 mM EDTA, 20 mM Tris- $\mathrm{HCl}$ pH-8.1, 500 $\mathrm{mM} \mathrm{NaCl}), \mathrm{LiCl}$ wash buffer $(0.25 \mathrm{mM} \mathrm{LiCl}, 1 \%$ IGEPALCA630, 1\% deoxycholic acid, $1 \mathrm{mM}$ EDTA, $10 \mathrm{mM}$ Tris-pH 8.1) and TE buffer. The immunocomplexes were eluted two times by adding a $250 \mathrm{ml}$ aliquot of a freshly prepared solution of $1 \% \mathrm{SDS}, 0.1 \mathrm{M} \mathrm{NaHCO}$ and the cross-linking reactions were reversed by incubation at $65^{\circ} \mathrm{C}$ for $4 \mathrm{~h}$. Further, the samples were digested with proteinase $\mathrm{K}(10 \mathrm{mg} / \mathrm{ml})$ at $42^{\circ} \mathrm{C}$ for $1 \mathrm{hr}$, DNA was recovered by phenol/chloroform extractions, ethanol precipitated using $1 \mathrm{ml}$ of $20 \mathrm{mg} / \mathrm{ml}$ glycogen as the carrier, and suspended in sterile water. For PCR analysis, aliquots of chromatin before immunoprecipitation were saved (Input). PCR was performed to analyse the presence of DNA precipitated by Runx 2 specific antibody, and by using specific primers to amplify a fragment of the Slug gene promoter.

Each PCR reaction was performed with $5 \mu$ l of the bound DNA fraction or $2 \mu \mathrm{l}$ of the input. The PCR was performed as follows: preincubation at $95^{\circ} \mathrm{C}$ for $5 \mathrm{~min}, 30$ cycles of 1 min denaturation at $95^{\circ} \mathrm{C}, 1 \mathrm{~min}$ annealing at $62^{\circ} \mathrm{C}$ and 1 min at $72^{\circ} \mathrm{C}$, with one final incubation at $72^{\circ} \mathrm{C}$ for $5 \mathrm{~min}$. No-antibody control was included in each experiment.

\section{Statistical analysis}

Data are presented as the mean \pm SEM calculated from at least three independent experiments performed on material obtained from all patient analysed. Statistical analysis was performed by one-way analysis of variance and the Student's t-test. A p-value $<0.05$ was considered statistically significant.

\section{RESULTS}

Isolation and characterization of human primary osteoblasts Human primary osteoblast cultures (hOBs) were generated from bone chips removed from nasal septum, and were subjected to the experimental procedure schematized in Figure 1. 
From a group of 81 patients, 10 samples have been selected for the analysis here described, essentially based on their ability to proliferate and differentiate. The appearance of isolated human osteoblastic cells was examined and we found cell morphology to be consistent with what has been reported in the literature (Figure 1) (22). It was observed that immediately following isolation, there was limited cellular migration from the trabecular bone chips at day 1 . After about 7 days, outgrowth of bone cells from the bone chips commenced, and confluency in T25 flask was usually reached after 3-4 weeks. The cellular morphology was spindle-shape as well as circular for some osteoblastic cells. On examination of the cells at day 21 , there was a substantial increase in the number of primary cells that had migrated from the bone chips, increasingly further away from the bone chips.

The immunophenotypical profile of the cells from all 10 samples was determined by flow cytometric analysis The results indicate that most of the cells $(98.12 \%)$ expressed cell surface marker CD90 (Thy-1) (Figure 2A) that is detected in the early stage of osteoblast differentiation and declines as osteoblasts differentiate into osteocytes ${ }^{(23)}$. The hematopoietic marker CD45 was not detectable on these cells (Figure 2A), indicating that cells were not contaminated with cells of hematopoietic origin. In addition, flow cytometry showed that the cells did not express STRO1 (Figure 2A), suggesting that they are mostly mature osteoblasts ${ }^{(24)}$. We then analysed the phenotypic markers by immunocytochemical staining. The cells from all 10 samples were highly positive for both Runx2, a typical osteogenic marker, and Slug, a zinc finger transcription factor involved in epithelial-mesenchymal transition and recently correlated with osteoblast maturation (Figure 2B) ${ }^{(20,25)}$. A significant component of the bone extracellular matrix, the bone

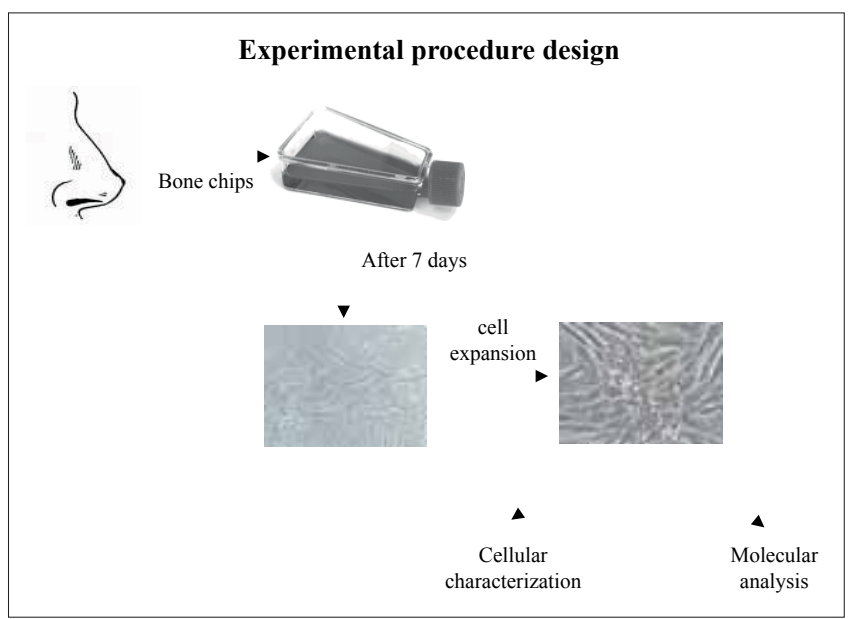

Figure 1. Isolation and use of human primary osteoblasts (hOBs). Human primary osteoblasts were obtained from bone chips removed from nasal septum. Bone was cut in small pieces which were rinsed and then cultured in T25 flask. About 7 days after isolation, outgrowth of bone cells from bone chips began. As shown in this figure, cells present a spindle-shape morphology. Once hOBs reached confluency, they were used for cellular and molecular analysis. sialoprotein (BSP), was detected at mRNA level by real time RT-PCR. These data confirm the level of maturation of the cells giving a definite indication of their commitment.

Next, the cells were characterized for their osteogenic capacity in terms of alkaline phosphatase (ALP) activity and mineral deposition (Figure 2C). The presence of sites of ALP activity was detected in all samples. In addition, Alizarin Red staining showed that all samples analysed exhibited an evident extracellular matrix mineralization after 14 days of culture under osteogenic conditions (a representative sample is shown in Figure 2C).

\section{Effect of a specific gene knockdown}

To analyse the potential to modify cellular phenotype in consequence of nucleic acid based drug treatments, 4 of the 10 samples were transfected with a double-stranded fluorescent dye-labeled oligonucleotide and the DNA uptake has been monitored for increasing length of time (2-24 hrs). After 24 hrs, the fluorescent molecules were widely distributed into the nuclei of most of the cells (Figure 3A), and the transfection efficiency was estimated about $80 \%$. Automated cell culture observation by using the BioStation CT-LA image analysis system confirmed the results (data not shown).

To determine the effect of a specific oligonucleotide treatment, the cells were then transfected with a siRNA against Slug, a transcription factor that we previously demonstrated correlated with osteoblastic phenotype ${ }^{(20)}$. The transfection significantly decreased the mRNA and protein levels of Slug, compared with those in cells transfected with scramble siRNA (Figure 3B). Concomitantly, the ability to deposit mineral matrix of the cells decreased significantly, demonstrating that the cells clearly respond to modulation of their osteoblast phenotype (Figure 3B).

Runx2 interacts "in vivo" with the promoter of SLUG gene To study the feasibility of other molecular investigations, 4 of the 10 samples were subjected to chromatin immunoprecipitation (ChIP) analysis, an "in vivo" transcriptional regulation assay. We set up sufficiently sensitive ChIP conditions to allow the analysis of primary cells. At this purpose, the involvement of a bone-specific transcription factor, Runx2, in the transcriptional regulation of SLUG, was tested. Using a program for predicting transcription factor binding sites (TFSEARCH, www.cbrc.jp/research/db/TFSEARCH.html), two potential cis elements for Runx 2 were found in the promoter of the human SLUG gene at -537 and -476 (Figure 4). Their functionality was investigated by ChIP assay, analysing the "in vivo" association between Runx 2 and the SLUG promoter. The cells were exposed to formaldehyde to cross-link proteins and DNA, and sonicated to fragment the chromatin. Specific antibody against Runx2 was used to immunoprecipitate the protein - DNA complexes, and Runx 2 recruitment was assessed by using the specific set of primers reported in Table 1. The presence of the promoter specific DNA region before immunoprecipitation 


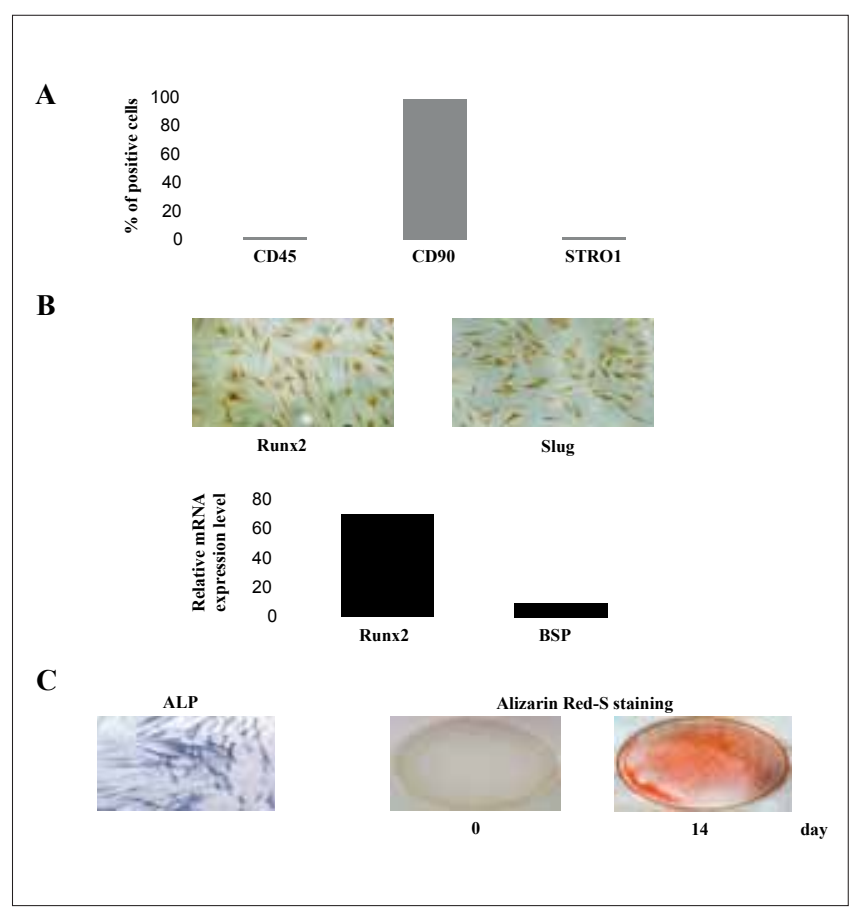

Figure 2. Phenotypical characterization of human primary osteoblasts (hOBs). A) The characterization of ten hOBs has been performed by flow cytometric analysis of CD45, CD90 and STRO-1 phenotypical markers. Data were expressed as \% positive cells \pm SEM, repeated three times for each sample. B) hOBs samples were subjected to immunocytochemical analysis for Runx2 and Slug phenotypical markers. A representative sample is shown (X10 magnification). The level of Runx2 and BSP expression was examined by quantitative RT-PCR in ten hOB samples. The experiments were carried out in triplicate, the expression levels were normalized on the basis of GAPDH expression and results of the experiments are reported as relative mRNA expression levels. $\Delta \Delta \mathrm{Ct}$ method was used to value the gene expression; SEM was calculated. C) The cells were treated with $\beta$-glycerophosphate, ascorbic acid, and dexamethasone and formation of extracellular matrix was valuated. Mineral formation was examined by Alizarin Red-S staining after 14 days of osteogenic induction. The authentic osteoblast phenotype was confirmed in hOBs by staining for alkaline phosphatase (ALP) activity.

was confirmed by PCR (input). After immunoprecipitation, DNA was extracted from the beads and used as a template to generate a specific PCR product spanning the putative Runx2 binding site in the SLUG promoter gene. As shown in Figure 4, we demonstrated that the SLUG gene displays a specific regulatory response in osteoblasts with the recruitment of a bone-specific transcription factor, Runx2, that is required for terminal differentiation of osteoblasts. As expected, no recruitment of this bone-specific transcription factor was observed in a sequence of SLUG promoter, (region 2), lacking of Runx2 binding sites (Figure 4) or in MCF-7 breast cancer cells (data not shown).

These ChIP experiments strengthen the role of Slug in osteoblasts showing that it is a Runx 2 target gene, and demonstrate the feasibility of this analysis in human primary osteoblasts obtained from nasal septum.

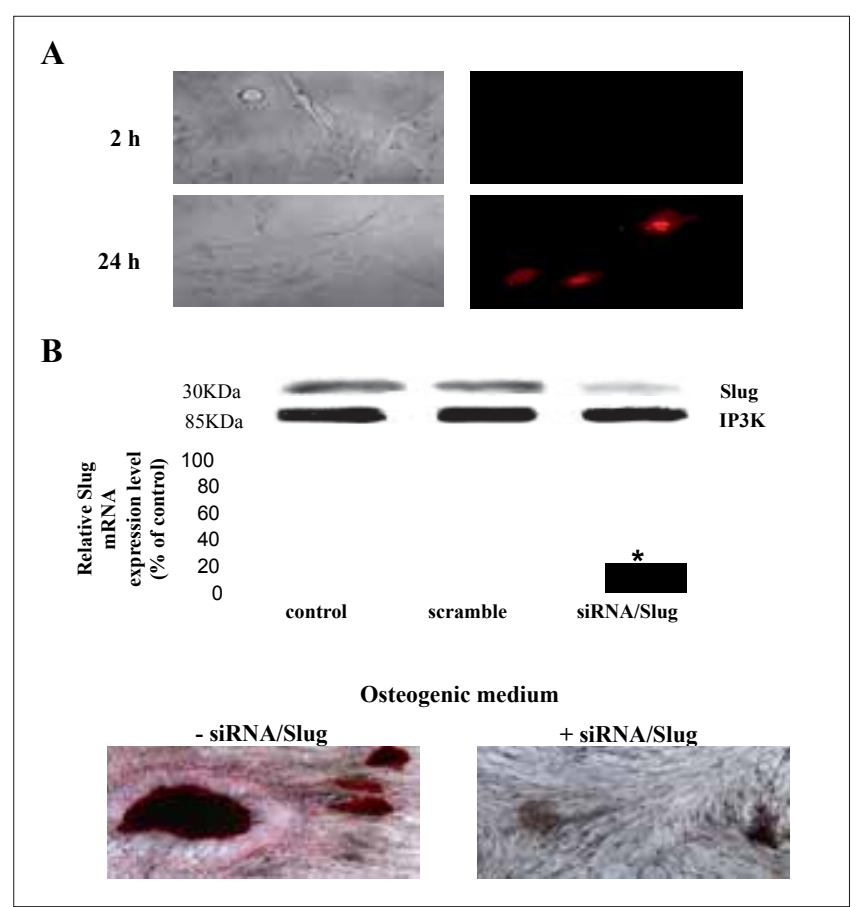

Figure 3. Transfection efficiency and effect of Slug knockdown during osteogenic differentiation. A) Cells were transfected with $30 \mathrm{nM}$ of a double-stranded fluorescent dye-labeled oligonucleotide. The uptake of oligo fluorescent siRNA was analysed in hOBs by fluorescence microscopy. Two and $24 \mathrm{hrs}$ after the transfection, cells were washed and examined under phase contrast (left panels) or fluorescent light using a fluorescent filter (right panels). B) hOBs were treated for 6 days with siRNA/Slug2 $30 \mathrm{nM}$ or a scramble siRNA as negative control. At the top, representative western blot of siRNA/Slug2 treated cells shows a specific decrease of endogenous Slug protein level. Size markers are reported (KDa). IP3K was used as a loading control. RT-PCR results, after correction to GAPDH content, are expressed as siRNA/ Slug2 over control ratio. Results represent means \pm SEM of 4 hOB samples $\left({ }^{*} p<0.05\right)$. After siRNA/Slug treatment, the cells cultured in osteogenic medium and analysed for the deposition of calcium salts by Alizarin Red-S staining. At day 14, more mineralized nodules were observed in untreated cells respect to silenced cells.

\section{DISCUSSION}

The tissue engineering supported by cell therapy and scaffolds with advanced carrier technology for growth factors offers important opportunity for bone tissue repair and regeneration. It may be based on different approaches that combine the collaboration between surgeons, scientists and engineers.

Table 1. PCR primers used for chromatin immunoprecipitation assay (ChIP).

\begin{tabular}{llr}
\hline Regions & Primer sequences & $\begin{array}{r}\text { Product } \\
\text { size (bp) }\end{array}$ \\
\hline Region 1 Forward F1: 5'-ATATAGGCTCTCATTAACAC-3', 218
\end{tabular}
Reverse R1: 5'-AGTATTTTCAAGAGAGGTAA-3'

Region 2 Forward F2: 5'-GAAATGGAGTGAAAAGCAAG-3' 175 Reverse R2: 5'-TTGCAGGAGAGAGGAAAATA-3' 


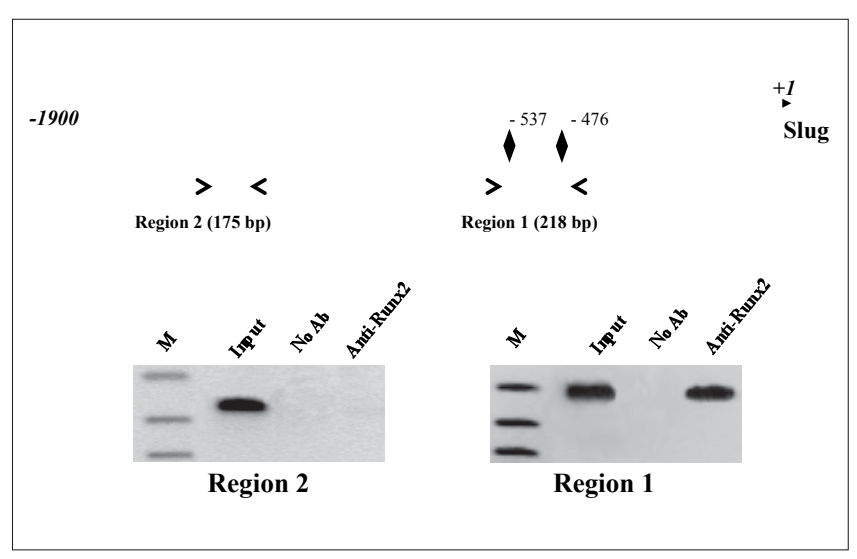

Figure 4. In vivo recruitment of Runx2 transcription factor on human Slug promoter. The Slug promoter region under investigation is reported $(+1 /-1900)$. The positions of the putative Runx2 consensus binding sites are enclosed by black diamonds. Recruitment of Runx2 transcription factor to the human Slug promoter is demonstrated by 'in vivo' ChIP binding assays. Protein-DNA complexes were formaldehyde-cross-linked in hOBs in vivo. Chromatin fragments from these cells were subjected to immunoprecipitation with antibody against Runx2. After cross-link reversal, the coimmunoprecipitated DNA was amplified by PCR using the reported primers, which positions are indicated with arrows. Region 2, lacking Runx2 binding sites, represents a negative control of ChIP experiments. PCR fragments were resolved through $1.5 \%$ agarose gels. No Ab represents a negative control. Input represents a positive control using the starting material $(0.2 \%)$ prior to immunoprecipitation. The molecular weights of PCR fragments are shown in parentheses. M: molecular weight marker.

Because the formation of bone tissue is based mainly on the action of osteoblasts, these cells are of special relevance (26). Formation of bone involves a complex pattern of cellular events initiated by proliferation and differentiation of mesenchymal precursor cells into bone-forming cells and finally resulting in the mineralization of the extracellular matrix. Therefore, the ultimate aim of skeletal tissue regeneration is mineral deposition in a scaffold with a pore system for growth of suitable cells, such stem cells or precursor cells that may be implanted or seeded into the scaffolds capable of supporting three-dimensional tissue formation ${ }^{(4-7,18,27,28)}$. Various scaffolds have already been developed for bone tissue engineering applications. However, the properties of cellular component are considered particularly determining factors.

Reconstructive strategies of the craniofacial district, naso-orbito-ethmoidal fractures, whether isolated or as a component of complex facial fractures, are always some of the most challenging skeletal facial injuries to treat ${ }^{(1-3,8-17,29-32)}$. Regeneration of bone tissue is also required after frequent maxillary or mandible reabsorption due to tumour resections or tissue necrosis. The successful facial plastic surgery approaches and the development of more appropriate and effective treatments are based on knowledge of bone biology including cellular and molecular aspects of bone development, formation and repair. It is important to underline that several clinical studies on congenital defects of nasal septum and the findings on experimental animals after nasal septum ablation demonstrate that the septum acts as a growth center thrusting the midface bones downwards and forwards from the cranial base.

Therefore, a search for the ideal cells to employ in facial skeleton regenerative medicine, brought us to consider the potential use of bone cells obtained from nasal septum for autologous reconstruction techniques. In fact, the recovery and propagation facility of these cells make them both a good alternative to bone marrow osteogenic progenitors from iliac crest, and qualified differentiated cells that could be directly applied to the bone defect in combination with suitable scaffolds. It is important to underline that the lower part of the osseous nasal septum that we used in this study is part of the maxilla, and it has been previously demonstrated that the use of osteoblasts of maxillary origin is safe ${ }^{(33)}$. We demonstrated in the present study that cells derived from explants of nasal septum are easy to obtain, can be grown up in culture, express markers of osteoprogenitor cells, and are committed towards maturation because do not express STRO1. In fact, previous studies have shown that the STRO1 antigen defines a MSC precursor subpopulation, is present in osteogenic precursor cells isolated from human bone marrow, is maintained in immature, pre-osteoblastic phenotype, and is progressively lost by mature osteoblasts ${ }^{(24)}$.

Under appropriate treatment, the cells can be induced to osteogenic differentiation giving rise to mature osteoblasts characterized by the expression of osteogenic markers, and ability to deposit mineralized matrix. These evidences indicate that these cells may represent a very promising material to be utilized in bone cellular therapy.

At the same time, we demonstrated that, from a single sample, it is possible to obtain a sufficient quantity of cells to perform several molecular analysis including RT-PCR, ChIP, and immunocytochemical experiments. The information that can be obtained from the molecular approaches described here are worthy of interest because biochemical signal cascades that alter expression of genes or activation of proteins play critical role in tissue engineering field. Many of these signals, only in part known, include FGF, WNT, TGF- , BMP, EGF, HIF, Notch, PTH, and integrins - mediated pathways, and are correlated with changes in specific activities of bone cells and may be also considered potential therapeutical targets ${ }^{(25,34)}$. Thus, it is very important, where feasible, to perform a direct molecular analysis on the same cells that are then to be used for tissue engineering.

All together, our findings may be considered a preliminary statement for clinical applications in the head and neck area aimed to develop an ex vivo model for induction of ectopic bone formation using autologous committed precursors cells. 


\section{ACKNOWLEDGEMENTS}

This research was supported by grants from Regione Emilia Romagna, Programma di Ricerca Regione Universita' 20072009. E.L. is a recipient of a fellowship from the Fondazione Cassa di Risparmio di Cento.

\section{REFERENCES}

1. Kuriakose MA, Shnayder Y, DeLacure MD. Reconstruction of segmental mandibular defects by distraction osteogenesis for mandibular reconstruction. Head \& Neck 2003; 25: 816-824.

2. Cordeiro PG. Frontiers in free flap reconstruction in the head and neck. J Surg Oncol. 2008; 97: 669-673.

3. Nussenbaum B, Rutherford RB, Krebsbach PH. Bone regeneration in cranial defects previously treated with radiation. Laryngoscope 2005: 115: 1170-1177.

4. Panetta NJ, Gupta DM, Longaker MT. Bone Regeneration and Repair. Curr Stem Cell Res Ther. 2010; 5: 122-128.

5. Porter JR, Ruckh TT, Popat KC. Bone tissue engineering: a review in bone biomimetics and drug delivery strategies. Biotechnol Prog. 2009; 25: 1539-1560.

6. Bueno EM, Glowacki J. Cell-free and cell-based approaches for bone regeneration. Nat Rev Rheumatol. 2009; 5: 685-697.

7. Grayson WL, Fröhlich M, Yeager K, et al. Regenerative medicine special feature: engineering anatomically shaped human bone grafts. Proc Natl Acad Sci USA. 2010; 107: 3299-3304.

8. Goessler UR, Stern-Straeter J, Riedel K, et al. Tissue engineering in head and neck reconstructive surgery: what type of tissue do we need? Eur Arch Otorhinolaryngol. 2007; 264: 1343-1356.

9. Bücheler M, Haisch A. Tissue engineering in otorhinolaryngology. DNA Cell Biol. 2003; 22: 549-564.

10. Malard O, Espitalier F, Bordure P, et al. Biomaterials for tissue reconstruction and bone substitution of the ear, nose and throat, face and neck. Expert Rev Med Devices 2007; 4: 729-739.

11. Pellegrini G, Seol YJ, Gruber R, et al. Pre-clinical models for oral and periodontal reconstructive therapies. J Dent Res. 2009; 88: 1065-1076.

12. Scheller EL, Krebsbach PH, Kohn DH. Tissue engineering: state of the art in oral rehabilitation. J Oral Rehabil. 2009: 36: 368-389.

13. Moioli EK, Clark PA, Xin X, et al. Matrices and scaffolds for drug delivery in dental, oral and craniofacial tissue engineering. Adv Drug Deliv Rev. 2007; 59: 308-324.

14. Gerressen M, Hermanns-Sachweh B, Riediger D, et al. Purely cancellous vs. corticocancellous bone in sinus floor augmentation with autogenous iliac crest: a prospective clinical trial. Clin Oral Implants Res. 2009; 20: 109-115.

15. Richmon JD, Sage AB, Shelton E, et al. Effect of growth factors on cell proliferation, matrix deposition, and morphology of human nasal septal chondrocytes cultured in monolayer. Laryngoscope 2005; 115: 1553-1560.

16. Rotter N, Haisch A, Bücheler M. Cartilage and bone tissue engineering for reconstructive head and neck surgery. Eur Arch Otorhinolaryngol. 2005; 262: 539-545.

17. Zaky SH, Cancedda R. Engineering craniofacial structures: facing the challenge. J Dent Res. 2009; 88: 1077-1091.

18. Khan SN, Cammisa, FP, Sandhu HS, et al. The biology of bone grafting. J Am Acad Orthop Surg. 2005; 13: 77-86.
19. Lambertini E, Franceschetti T, Torreggiani E, et al. Slug: a new target of lymphoid enhancer factor-1 in human osteoblasts. BMC Mol Biol. 2010; 11: 13.

20. Lambertini E, Lisignoli G, Torreggiani E, et al. Slug gene expression supports human osteoblast maturation. Cell Mol Life Sci. 2009; 66: 3641-3653.

21. Lambertini E, Tavanti E, Torreggiani E, et al. ERalpha and AP-1 interact in vivo with a specific sequence of the $\mathrm{F}$ promoter of the human ERalpha gene in osteoblasts. J Cell Physiol. 2008; 216: 101-110.

22. Robey PG, Termine JD. Human bone cells in vitro. Calcif Tissue Int. 1985; 37: 453-460.

23. Chen XD, Qian HY, Neff L, et al. Thy-1 antigen expression by cells in the osteoblast lineage. J Bone Miner Res. 1999; 14: 362375.

24. Byers RJ, Brown J, Brandwood C, et al. Osteoblastic differentiation and mRNA analysis of STRO-1-positive human bone marrow stromal cells using primary in vitro culture and poly (A) PCR. J Pathol. 1999; 187: 374-381.

25. Soltanoff CS, Yang S, Chen W, et al. Signaling networks that control the lineage commitment and differentiation of bone cells. Crit Rev Eukaryot Gene Expr. 2009; 19: 1-46.

26. Lian JB, Stein GS. Concepts of osteoblast growth and differentiation: basis for modulation of bone cell development and tissue formation. Crit Rev Oral Biol Med. 1992; 3: 269-305.

27. Sundelacruz S, Kaplan DL. Stem cell- and scaffold-based tissue engineering approaches to osteochondral regenerative medicine. Semin Cell Dev Biol. 2009; 20: 646-655.

28. Penolazzi L, Tavanti E, Vecchiatini R, et al. Encapsulation of mesenchymal stem cells from Wharton's jelly in alginate microbeads. Tissue Eng Part C Methods. 2010; 16: 141-155.

29. Risbud MV, Shapiro IM. Stem cells in craniofacial and dental tissue engineering. Orthod Craniofac Res. 2005; 8: 54-59.

30. Scheller EL, Krebsbach PH, Kohn DH. Tissue engineering: state of the art in oral rehabilitation. J Oral Rehabil. 2009: 36: 368-389.

31. Scheller EL, Krebsbach PH. Gene therapy: design and prospects for craniofacial regeneration. J Dent Res. 2009; 88: 585-596.

32. Shanti RM, Li WJ, Nesti LJ, et al. Adult mesenchymal stem cells: biological properties, characteristics, and applications in maxillofacial surgery. J Oral Maxillofac Surg. 2007; 65: 1640-1647.

33. Doglioli P, Scortecci G. Characterization of endosteal osteoblasts isolated from human maxilla and mandible: an experimental system for biocompatibility tests. Cytotechnology 1991; 7: 39-48.

34. Lian JB, Javed A, Zaidi SK, et al. Regulatory controls for osteoblast growth and differentiation: role of Runx/Cbfa/AML factors. Crit Rev Eukaryot Gene Expr. 2004; 14: 1-41.

Roberta Piva

Department of Biochemistry and Molecular Biology

Section of Molecular Biology

University of Ferrara

Via Fossato di Mortara, 74

44121 Ferrara

Italy

E-mail: piv@unife.it 